\title{
Carbon, Graphene and Graphene Oxide Quantum Dots for Analytical Biochemistry Research
}

\section{Edward Lai}

Department of Chemistry and Institute of Biochemistry, Carleton University, 1125 Colonel By Drive, Ottawa, ON K1S 5B6, Canada

*Corresponding author: Edward Lai, Department of Chemistry and Institute of Biochemistry, Carleton University, 1125 Colonel By Drive, Ottawa, ON K1S 5B6, Canada, Tel: 613-520-2600-3835; E-mail: EdwardLai@Cunet.Carleton.Ca

Rec date: Jul 30, 2016; Acc date: August 1, 2016; Pub date: August 4, 2016

Copyright: (c) 2016 Lai E, This is an open-access article distributed under the terms of the Creative Commons Attribution License, which permits unrestricted use, distribution, and reproduction in any medium, provided the original author and source are credited.

\section{Editorial}

Graphene quantum dots (GQDs) have emerged rapidly as a new class of attractive fluorescence nano-probes ever since they were made from coal in 2013 by the Rice University lab of chemist James Tour $[1,2]$. They offer 2-10 $\mathrm{nm}$ size, good quantum yield, high photostability, tunable photoluminescence, flexible molecular structure, easy functionalization, excellent bio-compatibility, stable dispersion in water, and facile hydrothermal synthesis. A method for forming GQDs included adding an organic starting material to a vessel and heating the material to within $20^{\circ} \mathrm{C}$ of its boiling temperature for a time no longer than ten minutes [3]. Their chemical inertness and low toxicity have triggered numerous studies on their unique properties in interdisciplinary science and engineering research over the last several years [4]. Different sized GQDs with a narrow size distribution could be obtained via gel electrophoresis of the crude GQDs prepared through a photo-Fenton reaction of graphene oxide [5]. It was illustrated that the photoluminesce emissions of the well-defined GQDs originated mainly from the peripheral carboxylic groups and conjugated carbon backbone planes. GQDs enhanced sulfur/sulfide utilization, proving that $\mathrm{C}-\mathrm{S}$ bonding could occur [6]. Graphene oxide quantum dots (GOQDs) exhibited photoluminescence color variation from orange-red to blue as their size was reduced, with electron transitions from the graphene $\pi^{*}$ orbital to the oxygen $n$ orbital [7]. The electronic properties and mechanisms involved in quantumconfined photoluminescence can be applied to research in analytical biochemistry. Chiral nanostructures can greatly enrich the chemical property of graphene-based materials to facilitate their applications in biology. Covalent attachment of $1 / \mathrm{d}$-cysteine moieties to GQDs led to their helical buckling due to chiral interactions at the edges [8]. Exposure of liver HepG2 cells to the 1/d-GQD stereoisomers revealed a noticeable difference in their toxicity. Molecular dynamics simulations demonstrated that d-GQDs have a stronger tendency to accumulate within the cellular membrane than l-GQDs.

A fluorometric sensing platform based on tyramine-functionalized GQDs was able to detect a spectrum of metabolites with high sensitivity and specificity, as demonstrated in multi-parametric blood analysis for cholesterol, glucose, l-lactate and xanthine [9]. This metabolite profiling technique could be developed for the diagnosis of metabolic disorders and associated diseases, such as diabetes, gout, hypertension, lactic acidosis and obesity. A highly sensitive fluorometric method was developed for inorganic pyrophosphatase activity detection based on the disaggregation and aggregation of GQDs [10]. Copper ions triggered the severe aggregation of GQDs with rich carboxyl groups, which resulted in effective fluorescence quenching. With the addition of pyrophosphate, the quenched fluorescence was effectively recovered owing to its strong interaction with $\mathrm{Cu}^{2+}$. Under catalytic hydrolysis by pyrophosphatase, the pyrophosphate- $\mathrm{Cu}^{2+}$ complex was rapidly disassembled and the GQDs fluorescence was re-quenched. This method is highly sensitive and selective for the detection of pyrophosphatase, with a detection limit down to $1 \mathrm{mU} \mathrm{mL} \mathrm{mL}^{-1}$. One potential application is the diagnosis of pyrophosphatase-related diseases and screening of pyrophosphatase inhibitors in biological systems.

Carboxylic carbon quantum dots (cCQDs) functioned as a nanoquencher in the detection of nucleic acid based on a homogeneous fluorescent assay [11]. The principle lied in the different propensity of single-stranded DNA and double-stranded DNA to adsorb onto the surface of cCQDs. A superior range of detection, at least 3 orders of magnitude, was achieved; the potential to distinguish single-base mismatch was also exhibited. This may meet the high demand for simple, sensitive and selective DNA biosensors, particularly in disease diagnostics, environment monitoring, and food safety. A sensor for the detection of specific microRNA sequences was developed by exploiting mainly the $\pi-\pi$ stacking interaction between the sp2 carbon atoms of GQDs and the nucleobases of single-stranded DNA anchored on upconversion nanoparticles to enhance the upconversion emission [12]. Hybridization of the single-stranded DNA chains with their complementary microRNA sequences blocks the capacity of the upconversion nanoparticles to interact with the GQDs. That resulted in a reduction of the fluorescent enhancement, depending on the concentration of microRNA sequences down to a detection limit of 10 $\mu \mathrm{M}$. A dual-emission and two-photon GQDs probe for bioimaging of hydrogen peroxide in biological systems [13]. A boronate merocyanine fluorophore was used as both a target-activated trigger and the dualemission fluorescence modulator. Their fluorescence response is rapid and specific for mapping the production of hydrogen peroxide in living cells and deep tissues, with a quantitative detection limit of $0.05 \mu \mathrm{M}$.

GQDs were modified by grafting hexylamine or oleylamine to generate surfactant behavior [14]. Precise control of interfacial activity was demonstrated by varying the alkylamine density on the GQDs. The excellent tunability of GQDs' surface property highlights their potential use as a surfactant with novel functionality for stabilizing colloidal nanoparticles in analytical biochemistry research experiments. GQDs were used as an immobilized template on screen printed electrodes for the construction of a sensor platform based on electrochemical impedance spectroscopy $[15,16]$. The GQDs-modified electrode was conjugated with highly specific anti-myoglobin antibodies for the sensitive detection of myoglobin, a cardiac biomarker, in the presence of other competing proteins. The limit of detection was $0.01 \mathrm{ng} / \mathrm{mL}$ which is comparable to standard ELISA techniques. GQDs were added in a capillary electrophoresis method for the separation of cinnamic acid and its derivatives with direct UV detection. Excellent separation was achieved in eighteen minutes, 
Page 2 of 2

mainly attributing to the interaction of GQDs with isoferulic acid, sinapic acid and cinnamic acid.

Quantum dots have been used in food science mostly as a marker to either detect pathogenic bacteria or track proteins in the food matrix to give a better understanding of their role [17]. The most widely used quantum dots consist of a CdSe core functionalized in different ways and coated with polymers for better attachment to bacteria and proteins. The strong luminescent property of GQDs can be utilized to stain bacterial cells via a facile translocation into the cytoplasm [18]. In vitro experiments showed that GQDs exhibited very low cytotoxicity owing to their ultra-small size and high oxygen content; in vivo bio-distribution experiment of GQDs revealed no accumulation in the main organs of mice even after multiple doses and fast clearance through the kidneys [19]. The lack of obvious toxicity results obtained so far shows a promising future for more GQD applications in the food industry. Carbon dots are carbon nanoparticles that have very interesting optical properties and photocatalytic functions. They were highly effective in killing bacteria with visible light illumination, even under room lighting conditions [20]. These photoinduced bactericidal functions can provide many opportunities in a variety of bacteria control applications.

\section{References}

1. Rice University (2015) Fine-tuning quantum dots from coal. ScienceDaily, March 18.

2. Ye R, Peng Z, Metzger A, Lin J, Mann JA, et al. (2015) Bandgap engineering of coal-derived graphene quantum dots. ACS Appl Mater Interfaces 7: 7041-7048.

3. Zhao JX, Wu X (2015) Graphene quantum dots and method of making. Patents US20150284318 A1.

4. Shen J, Zhu Y, Yang X, Li C (2012) Graphene quantum dots: emergent nanolights for bioimaging, sensors, catalysis and photovoltaic devices. Chem Commun 48: 3686-3699.

5. Zhang F, Liu F, Wang C, Xin X, Liu J, et al. (2016) Effect of lateral size of graphene quantum dots on their properties and application. ACS Appl Mater Interfaces 8: 2104-2110.

6. Park J, Moon J, Kim C, Kang JH, Lim E, et al. (2016) Graphene quantum dots: structural integrity and oxygen functional groups for high sulfur/ sulfide utilization in lithium sulfur batteries. NPG Asia Materials 8: e272.

7. Yeh TF, Huang WL, Chung CJ, Chiang IT, Chen LC, et al. (2016) Elucidating confinement in graphene oxide dots based on excitation- wavelength-independent photoluminescence. J Phys Chem Lett 7: 2087-2092.

8. Suzuki N, Wang Y, Elvati P, Qu ZB, Kim K, et al. (2016) Chiral graphene quantum dots. ACS Nano 10: 1744-1755.

9. Li N, Than A, Wang X, Xu S, Sun L, et al. (2016) Ultrasensitive profiling of metabolites using tyramine-functionalized graphene quantum dots. ACS Nano 10: 3622-3629.

10. Zhu X, Liu J, Peng H, Jiang J, Yu R (2016) A novel fluorescence assay for inorganic pyrophosphatase based on modulated aggregation of graphene quantum dots. Analyst 141: 251-255.

11. Lo AH, Sofer Z, Bouša D, Ulbrich P, Bonanni A, et al. (2016) Carboxylic carbon quantum dots as a fluorescent sensing platform for DNA detection. ACS Appl. Mater. Interfaces 8: 1951-1957.

12. Laurenti M, Paez-Perez M, Algarra M, Alonso-Cristobal P, Lopez-Cabarcos E, et al. (2016) Enhancement of the upconversion emission by visible-tonear-infrared fluorescent graphene quantum dots for miRNA detection. ACS Appl Mater Interfaces 8: 12644-12651.

13. Zhao W, Li Y, Yang S, Chen Y, Zheng J, et al. (2016) Target-activated modulation of dual-color and two-photon fluorescence of graphene quantum dots for in vivo imaging of hydrogen peroxide. Anal Chem 88: 4833-4840.

14. Yang H, Ku KH, Shin JM, Lee J, Park CH, et al. (2016) Engineering the shape of block copolymer particles by surface-modulated graphene quantum dots. Chem Mater 28: 830-837.

15. Tuteja SK, Chen R, Kukkar M, Song CK, Mutreja R,et al. (2016) A label-free electrochemical immunosensor for the detection of cardiac marker using graphene quantum dots. Biosens Bioelectron 86: 548-556.

16. Sun Y, Bi Q, Zhang X, Wang L, Zhang X, et al. (2016) Graphene quantum dots as additives in capillary electrophoresis for separation cinnamic acid and its derivatives. Anal Biochem 500: 38-44.

17. Bonilla JC, Bozkurt F, Ansari S, Sozer N, Kokini JL (2016) Applications of quantum dots in food science and biology. Trends Food Sci Technol 53: 75-89.

18. Wang Z, Yu J, Zhang X, Li N, Liu B, et al. (2016) Large-scale and controllable synthesis of graphene quantum dots from rice husk biomass: a comprehensive utilization strategy. ACS Appl Mater Interfaces 8: 14341439 .

19. Chong Y, Ma Y, Shen H, Tu X, Zhou X, et al. (2014) In vitro and in vivo toxicity of graphene quantum dots. Biomaterials 35: 5041-5048.

20. Meziani MJ, Dong X, Zhu L, Jones LP, LeCroy GE, et al. (2016) Visiblelight-activated bactericidal functions of carbon quantum dots. ACS Appl Mater Interfaces 8: 10761-10766. 